\section{EARLY ENDOTHELIUM-DEPENDENT VASODILATORY DYSFUNCTION INNORMOTENSIVE, INTRA-UTERINE GROWTH RESTRICTED RATS IS RESTORED BY L-ARGININE AND INHIBITION OF ARGINASES}

\author{
I. Grandvuillemin ${ }^{1}$, I. Ligi ${ }^{1}$, C. Buffat ${ }^{1}$, F. Boubred ${ }^{1}$, \\ F. Dignat-George ${ }^{2}$, U. Simeoni ${ }^{1}$
}

${ }^{1}$ Department of Neonatology and INSERM UMR 608, '2INSERM UMR 608, Méditerannée University, Marseille, Marseille, France

Increasing clinical and experimental evidence demonstrates that fetalprogrammed hypertension in adulthood is associated with altered endotheliumdependent vasodilation. However, it is debated whether decreased endothelium-dependent vasodilation precedes or is a consequence of elevated arterial pressure. We studied endotheliumdependent vasodilating capacity and the L-argininenitric oxide pathway in intra-uterine growthrestricted (IUGR), 5-week-old, male rats, before the development of elevated arterial pressure. We furthermore tested the hypothesis that alteration in endothelial vasodilation may be due to a reduction in L-arginine bioavailability. Methods- Pregnant rats were fed either a control or protein restricted diet during gestation. Endothelium-intact aortic rings of 5-week-old male offspring of the control and IUGR groups were isolated and preconstricted with phenylephrine (PE) to test vascularreactivity to acetylcholine (Ach) and sodium nitroprusside (SNP), respectively endothelium-dependent and -independent vasodilators. Other experiments were conducted with L-arginine supplementation or with S-(2-boronoethyl)-L-cysteine (BEC), a specific endothelial arginase inhibitor. Arginase enzymatic activity was measured in aorta. ResultsSystolic blood pressure was not different between groups, and increased later in IUGR rats. While the vasodilatory response to SNP was similar in the two groups, a decreased vasodilatory response to Ach $(-20 \%, p<0.05)$ was observed in the IUGR group. Moreover, L-arginine supplementation and arginases inhibition restored the altered vasodilating capacity of aortic rings of IUGR animals. Arginase activity is enhanced in aorta of young IUGR rats. Conclusions- Reduced endothelium-dependent vascular relaxation precedes the developmentof fetal programmed hypertension in IUGR rats and involves the L-arginine-NO pathway. L-arginine supplementation and inhibition of arginases restore endothelium-dependent vasodilation in IUGR, prehypertensive rats.

\section{ORAL IODINE SUPPLEMENTATION AND THYROID FUNCTION IN PREMATURE BABIES BORN LESS THAN 1500 GR OF WEIGHT}

\section{S. Ares ${ }^{1}$, J. Arnaez¹, S. Santacruz², J. Quero1,} G. Morreale de Escobar ${ }^{3}$

${ }^{1}$ Neonatology, ${ }^{2}$ Biochemistry, University Hospital La Paz, ${ }^{3}$ Biochemistry, Instituto de Investigaciones Biomedicas, CSIC, Madrid, Spain

Background: The trace element iodine is essential for the synthesis of thyroid hormones. Premature babies need $>30 \mu \mathrm{g} \mathrm{I} / \mathrm{kg} /$ day (ICCIDD recommendations). Neonates and especially preterm infants are a population at risk of suffering the consequences of iodine deficiency, because of the impact of neonatal hypothyroxinemia on brain development.

Aims: oral lodine supplementation in very premature babies during the neonatal period.

Methods: Intervention group: we administered 30 $\mu \mathrm{g} \mathrm{I} / \mathrm{kg} /$ day of iodine in oral drops to 47 infants. Control group: 47 infants without supplements. The study was approved by the Ethics Committee. Samples of different formulas, maternal milk was kept for the determination of the iodine content.

Results: lodine content of mothers' breast milk: 15 (5.0) $\mu \mathrm{g} \mathrm{I} / \mathrm{dL}$ (range 1-60); Infants in the supplemented group reached the recommendations from the first days of life. Infants in the control group did not reach the recommended intake of iodine to 60 days of life. We found a positive relation between iodine intake and the concentrations of thyroid hormones until 60 days of life.

Conclusions: Thyroid function is related to iodine intake in preterm infants. Preterm babies on formula preparations and with parenteral nutrition are at high risk of iodine deficiency. Therefore, supplements should be added if iodine intake is found to be inadequate. Breast milk appears to be the best source of iodine for the premature infant.

Acknowledgments: We are grateful to the Fondo de Investigaciones Sanitarias (Grant 06/1310 for financial support). 